\section{APL approximations for common statistical tables}

\section{SELBY EVANS and LYNNE GILFILLAN \\ Texas Christian University, Fort Worth, Texas}

Statistical work makes frequent use of common statistical tables-the normal distribution, the $F$ and $t$ distributions, the chi-square distribution, and the binomial. In using a computer for statistical work, it is often convenient to have approximations to these tables available for immediate results or for use by other programs. Many APL libraries on large computers include functions to supply these approximations. Users of APL on microcomputers also need them. In this report, we present APL functions to meet that need.

The functions are presented in Figure 1. They are written and tested in APL*PLUS/PC, a version marketed by STSC, Inc., for the IBM PC and compatible machines. The functions, however, use no special features except the diamond, and they should run on any complete version of APL.

For IBM APL or other versions that may not accept the diamond, each expression set off by diamonds can be entered as a separate line. Take the expressions in order from the left.

The execution times given here were obtained from an IBM PC with the 8087 floating point coprocessor.

The Normal Distribution. The function ZTAB accepts a list of one or more variates in its right argument and returns a list carrying the approximate probability of obtaining a value less than or equal to each given variate, assuming a normal distribution. The sign of the variate is used. Thus the result for $-1.96,0,1.96$ is $.025, .5$, .975 .

ZTAB uses the approximation given by Zelen and Severo (1965) in Expression 26.2.17. A recent assessment of this and other approximations to the normal distribution (Brophy, 1983) suggests that it is a good choice.

Execution time for 100 variates is about $4 \mathrm{sec}$.

The $F$ and $t$ Tables. FTAB accepts degrees of freedom in its left argument and the corresponding $F$ ratios in its right argument. It returns the approximate probability of obtaining a value as great as or greater than the given value. Unlike ZTAB, FTAB returns the probability associated with the upper tail of the distribution because that is the only probability of interest.

Degrees of freedom can be given as a two-element vector or as an $N \times 2$ array if $N$ values are supplied in the right argument. Degrees of freedom for the numerator are given as the first element or first column. The order-

The authors' mailing address is: Psychology Department, Texas Christian University, Fort Worth, TX 76129. ing of the results, of course, matches the ordering of the inputs.

The approximation used by FTAB is given by Zelen and Severo (1965) in Expression 26.6.15. According to Kelley (1947), this approximation is good if the degrees of freedom for the denominator are greater than 3. Recommendations by Kelley (1947) and by Jaspen (1965) favor a correction for cases that fail to meet this condition. The correction is included in FTAB. (See Jaspen for the correct form of the expression.)

Table 1 presents illustrative results for small degrees of freedom. The test values in Table 1 are the $5 \%$ and $1 \%$ critical values taken from a standard table of $F$. The approximation appears to be adequate for tests at the .05 and .01 levels, assuming that the results are rounded off to hundredths before they are reported by the calling program.

Execution time for 100 variates is about $6 \mathrm{sec}$.

No function for approximating $t$ is needed, since the square of $t$ with $K$ degrees of freedom is distributed as $F$ with 1 and $K$ degrees of freedom. For a one-tailed test, divide the result by 2 .

The Chi-Square Table. CHISQ accepts degrees of freedom in its left argument and chi-square values in its right argument. The result carries the probability of obtaining a value equal to or greater than the given value under the assumed chi-square distribution. The left argument carries degrees of freedom and can be a scalar or a vector. The right argument carries one or more values to be tested. The arguments must match in ordering of elements; the result matches this ordering.

The procedure used by CHISQ is given by Zelen and Severo (1965) in Expressions 26.4.4, 26.4.5, and 26.4.14. The first two expressions are used with degrees of freedom of 30 or less. With degrees of freedom greater than 30 , the third expression is used. With multiple values in the arguments of CHISQ, large and small samples can be intermixed.

Execution time for 100 variates is $30-45 \mathrm{sec}$, depending on the mix of small and large samples.

The Binomial Distribution. BINOM accepts a frequency in its right argument and a number of trials in its left argument. It returns the probability of obtaining a frequency equal to or less than the given frequency, assuming the probability of the counted event on any given trial is .5. The right argument can carry multiple frequencies, but the left argument (the number of trials) must be a single value. To get the complete cumulative distribution for $N$ trials, enter 0 ,iota $\mathrm{N}$ as the right argument.

The procedure used by BINOM for sample size up to 150 is the standard combinatorial formula found in most statistical texts. It is not an approximation. For sample sizes greater than 150, the approximation to the normal distribution, also found in most statistical texts, is used. 


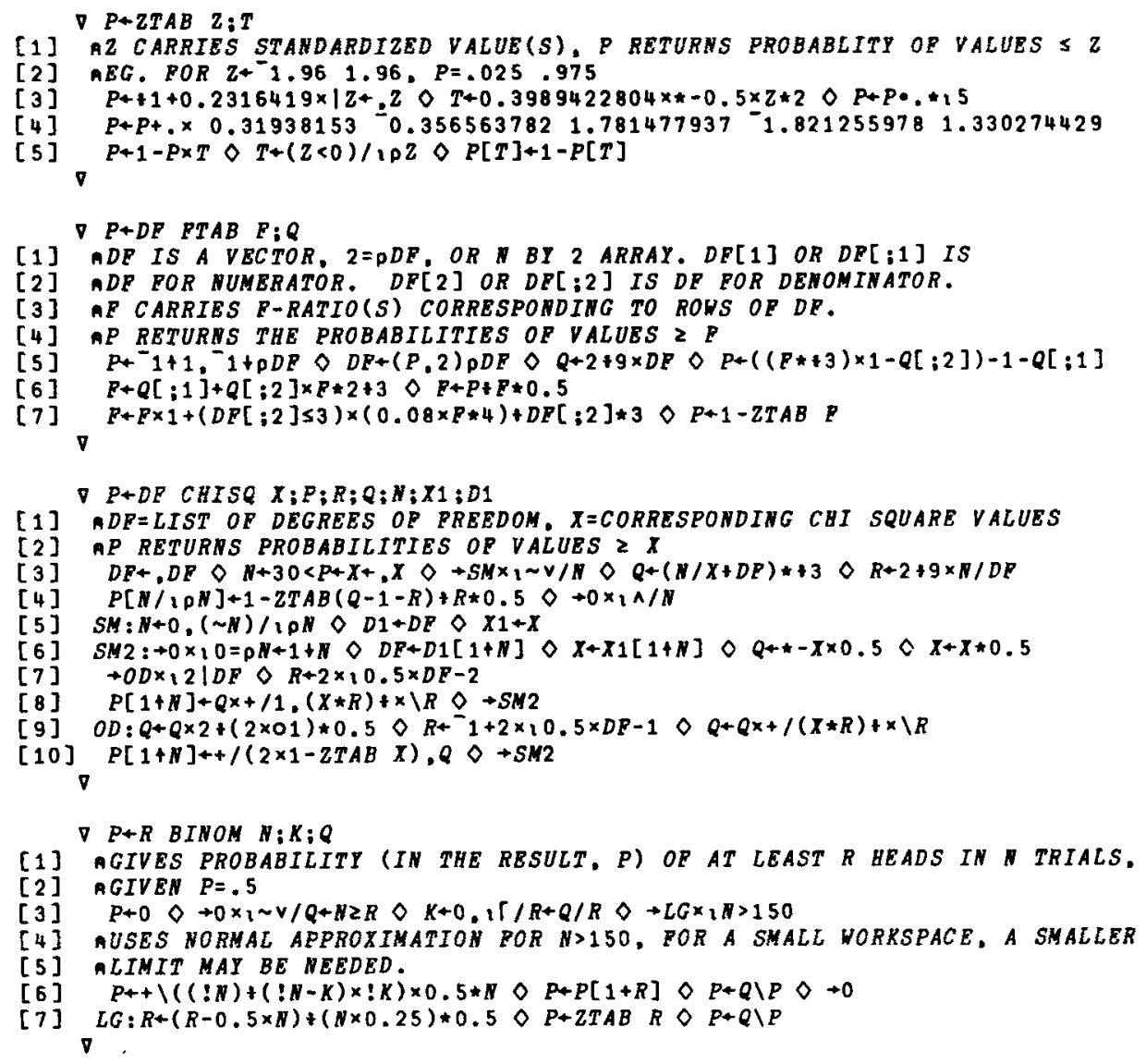

Figure 1. APL functions for approximate probabilities for the normal, $F$, chi-square, and binomial distributions.

Table 1

Probabilities Computed by FTAB for Selected Degrees of Freedom and F-Ratios at Probabilities of .05 and .01

\begin{tabular}{ccrrrc}
\hline DF1 & DF2 & $F, .05$ & $p$ & $F, .01$ & $p$ \\
\hline 1 & 1 & 161.00 & 0.049 & 4052.00 & 0.012 \\
1 & 2 & 18.51 & 0.047 & 98.49 & 0.007 \\
1 & 3 & 10.13 & 0.049 & 34.12 & 0.009 \\
1 & 4 & 7.71 & 0.050 & 21.20 & 0.011 \\
1 & 5 & 6.61 & 0.049 & 16.26 & 0.011 \\
2 & 2 & 19.00 & 0.049 & 99.00 & 0.008 \\
2 & 3 & 9.55 & 0.050 & 30.82 & 0.009 \\
2 & 4 & 6.94 & 0.051 & 18.00 & 0.012 \\
2 & 5 & 5.79 & 0.051 & 13.27 & 0.011 \\
3 & 3 & 9.28 & 0.050 & 29.46 & 0.010 \\
3 & 4 & 6.59 & 0.052 & 16.69 & 0.012 \\
3 & 5 & 5.41 & 0.051 & 12.06 & 0.011 \\
4 & 4 & 6.39 & 0.052 & 15.98 & 0.012 \\
4 & 5 & 5.19 & 0.051 & 11.39 & 0.011 \\
5 & 5 & 5.05 & 0.051 & 10.97 & 0.012 \\
\hline
\end{tabular}

Execution time for all 61 possibilities in a sample size of 60 is about $5 \mathrm{sec}$.

\section{REFERENCES}

Brophy, A. L. (1983). Accuracy and speed of seven approximations of the normal distribution function. Behavior Research Methods \& Instrumentation, 15, 604-605.

JASPEN, N. (1965). The calculation of probabilities corresponding to values of $z, t, F$, and chi-square. Educational \& Psychological Measurement, 25, 877-880.

KelleY, T. L. (1947). Fundamentals of statistics. Cambridge: Harvard University Press.

Zelen, M., \& Severo, N. C. (1965). Probability functions. In M. Abramawitz \& I. A. Stegun (Eds.), Handbook of mathematical functions. New York: Dover.

(Manuscript accepted for publication April 8, 1986.) 